Mathematical Research Letters 1, 631-638 (1994)

\title{
HARMONIC MAPS OF INFINITE ENERGY AND RIGIDITY RESULTS FOR QUASIPROJECTIVE VARIETIES
}

\author{
JÜRGEN JOST AND KANG ZUO
}

\section{Introduction}

Hodge theory is a fundamental tool of Kähler geometry. It represents cohomology classes by harmonic forms, and deriving properties of these harmonic forms then in turn yields information about the cohomology. Homology or cohomology groups, however, contain only partial information about the topology of a manifold. The most important topological invariant of a manifold $X$ is the fundamental group $\pi_{1}(X)$. Via the Albanese period mapping, $\pi_{1}(X)$ acts as a lattice on some vector space. We thus have an abelian representation of $\pi_{1}(X)$ yielding the first homology group. In order to obtain further information about the topology via $\pi_{1}(X)$, it is then natural to also study nonabelian representations of $\pi_{1}(X)$. In the same way as the Albanese period map is harmonic (this is essentially the Hodge theorem for 1-forms), for a nonabelian representation, one tries to construct an equivariant harmonic map $u$ and to study its properties. In the case of a compact Riemannian, Kählerian, or algebraic manifold, this theory is already well developed and too extensive to be reviewed here.

Many manifolds naturally arising in geometry, however, are noncompact. Here, we want to discuss the case of quasiprojective manifolds, i.e. those that can be compactified by adding a divisor at infinity. The local topology around infinity may be quite intricate, and much of it gets lost when passing to the compactification. Iitaka [I] extended the Albanese map to quasiprojective varieties. The harmonic map theory was extended to the quasiprojective case by Jost-Yau [JY2], [JY3], and Jost-Zuo [JZ1], [JZ2]. These papers, however, deal with a situation where one can construct a harmonic map of finite energy, and this requires some additional assumptions about the representation of $\pi_{1}(X)$ near infinity. In the onedimensional case, harmonic maps of possibly infinite energy have been produced and used by Simpson [S1], and in a different context by Wolf

Received August 30, 1994.

Research supported by SFB 237 and the Leibniz Program of the DFG. 
[W] and Lohkamp [Lk]. There were two interconnected reasons for working with finite energy maps. The first one is that their existence can be shown by a variational method. The second one is that they can be well enough controlled in order to be able to integrate Bochner type identities with finite results that allow one to deduce the vanishing of the individual terms because of sign conditions resulting from curvature assumptions. In particular, in the context where the theory is applied in Kähler geometry, such maps turn out to be pluriharmonic. This means that their restriction to any complex subvariety is again harmonic. This condition in fact is independent of the particular Kähler metric chosen on the domain, and it gives rise to a certain holomorphic foliation of the domain first discovered in [JY1]. Under additional assumptions, Siu's Bochner identity [Su1] even implies that the map is \pm holomorphic. From these two facts, one then deduces rigidity results that will be described in more detail below.

The main technical achievement of our work that is described in this note is an existence result for pluriharmonic maps of possibly infinite energy. This result will be described in $\S 1$. In $\S 2$, we complete the harmonic map approach to Margulis superrigidity for lattices in bounded symmetric domains. In $\S 3$, we present results on the factorization through lower dimensional varieties of archimedean and $p$-adic representations of fundamental groups of quasiprojective manifolds.

Detailed proofs of our results will appear elsewhere [JZ3].

\section{Existence of pluriharmonic maps}

Theorem 1. Let $X$ be a smooth quasiprojective variety. Let $Y$ be either a symmetric space of noncompact type or a locally compact Euclidean Tits building (with isometry group operating transitively on the set of vertices). Let

$$
\rho: \pi_{1}(X) \rightarrow I(Y)
$$

be a reductive (see below) homomorphism into the isometry group of $Y$. Then there exists a $\rho$-equivariant pluriharmonic map

$$
u: \tilde{X} \rightarrow Y
$$

from the universal cover of $X$ to $Y$ that satisfies the following estimate: Let $\bar{\Sigma}$ be a holomorphic curve in $\bar{X}$ with $\bar{\Sigma} \cap \bar{X} \backslash X=\left\{p_{0}\right\}$ locally. We represent a neighborhood of the node $p_{0}$ in $\Sigma:=\bar{\Sigma} \backslash\left\{p_{0}\right\}$ conformally as

$$
D^{*}:=\{z \in \mathbb{C}: 0<|z|<1\} .
$$


W.r.t. the (noncomplete) Euclidean metric on $D^{*}$ and the natural metric on $Y$ for the norm of the derivative on $u$, we then have:

$$
\|d u(z)\|^{2} \leq \frac{\text { const }}{|z|^{2}}
$$

(If $Y$ is a Tits building, $u$ is only Lipschitz, and the preceding estimate and the pluriharmonicity of $u$ have to be interpreted in the sense of Gromov-Schoen [GS].)

The following definition was suggested by Scot Adams.

Definition. Let $Y$ be a complete, simply connected, locally compact space of nonpositive curvature with isometry group $I(Y)$. Let $\Gamma$ be a group, $\rho: \Gamma \rightarrow I(Y)$ a homomorphism. $\rho$ is called reductive if there exists a complete totally geodesic subspace $Z$ of $Y$ stabilized by $\rho(\Gamma)$ with the following property:

For every totally geodesic subspace $Z^{\prime}$ of $Z$ that has no Euclidean factor, $\rho(\Gamma)$ does not fix any point in the sphere at infinity of $Z^{\prime}$, i.e. is not contained in a parabolic subgroup of the isometry group of $Z^{\prime}$.

In geometric terms, this means that there does not exist an unbounded sequence $\left(y_{n}\right)_{n \in \mathbb{N}} \subset Z^{\prime}$ with

$$
\operatorname{dist}\left(y_{n}, \gamma\left(y_{n}\right)\right) \leq c(\gamma)
$$

for all $\gamma \in \rho(\Gamma)$, with a constant depending on $\gamma$, but not on $n$. Semisimple representations are reductive.

For simplicity of notation, we shall often consider $u$ as a map from $X$ into $N:=Y / \rho\left(\pi_{1}(X)\right)$ although $N$ may be singular. Energy functionals $E$ can be computed on a fundamental region for $X$ in $\tilde{X}$.

We now outline the proof of Theorem 1:

We denote the distance function on $Y$ by $d(\cdot, \cdot)$. We assume here that all elements $g$ of $\rho\left(\pi_{1}(X)\right)$ are hyperbolic or elliptic, i.e. that

$$
\inf _{y \in Y} d(y, g y)
$$

is achieved. If this infimum is not achieved then our subsequent estimates will hold with some additional error term. This term is bounded because of the special geometry of our target (for example, on symmetric spaces all Jacobi fields decay exponentially). This in fact is the reason why our constructions do not work for more general target spaces of nonpositive curvature. 
Our proof proceeds by an induction on the dimension of $X$. We therefore first recall the analysis of Wolf [W] and Lohkamp [Lk] for complex dimension 1 . We let $\Sigma$ be a curve with a puncture. The analysis is local near the puncture, and we represent a neighborhood of the puncture conformally as

$$
A:=[0, \infty) \times S^{1}
$$

For $r>0$,

$$
A_{r}:=[0, r] \times S^{1} \subset A, \quad \Sigma_{r}:=(\Sigma \backslash A) \cup A_{r} .
$$

By our above assumption, under $\rho$, the curves $\{t\} \times S^{1}$ correspond to an elliptic or hyperbolic element, and so the image can be deformed to a point or a closed geodesic $\gamma$ in $N$. We map $S^{1}$ onto $\gamma$ proportionally to arclength via a map $g_{\gamma}$. There then exists a harmonic map

$$
u_{r}: \Sigma_{r} \rightarrow N
$$

with

$$
\left.\left.u_{r}\right|_{\partial \Sigma_{r}}=g_{\gamma} \quad \text { (identifying } \partial \Sigma_{r}=\{r\} \times S^{1} \text { with } S^{1}\right),
$$

since $\Sigma_{r}$ is compact. We want to obtain the desired harmonic map

$$
u: \Sigma \rightarrow N
$$

as $\lim _{r \rightarrow \infty} u_{r}$. In order to show the existence of this limit, the maps $u_{r}$ need to satisfy estimates on $\Sigma_{s}$ for fixed $s$ that are independent of $r$ as $r \rightarrow \infty$. For that purpose, we construct a comparison map

$$
v: \Sigma \rightarrow N
$$

by

$$
v_{\left.\right|_{A}}=g_{\gamma} \cdot \pi,
$$

where $\pi: A \rightarrow S^{1}$ is the obvious projection, and extending $v$ to all of $\Sigma$ as a Lipschitz map in the homotopy class determined by $\rho$. With $v_{r}:=\left.v\right|_{\Sigma_{r}}$, $\kappa_{0}:=E\left(\left.v\right|_{\Sigma \backslash A}\right)(E=$ energy $), \ell(\gamma):=$ length of $\gamma$

$$
\begin{array}{rlr}
E\left(u_{r}\right) \leq & E\left(v_{r}\right) \quad \text { since } u_{r} \text { is energy minimizing } \\
& =E\left(\left.v_{r}\right|_{\Sigma_{s}}\right)+E\left(\left.v_{r}\right|_{A_{r} \backslash A_{s}}\right) & \\
\leq E\left(\left.v_{r}\right|_{\Sigma_{s}}\right)+E\left(\left.u_{r}\right|_{A_{r} \backslash A_{s}}\right) & \begin{array}{l}
\text { since one easily verifies that } \\
v_{r} \text { is absolutely energy min- } \\
\text { imizing in its free homotopy } \\
\text { class on } A_{r} \backslash A_{s}
\end{array} \\
& &
\end{array}
$$


This implies

$$
E\left(\left.u_{r}\right|_{\Sigma_{s}}\right) \leq \kappa_{0}+\frac{1}{2} s \ell(\gamma)^{2}
$$

which is independent of $r$ as desired. This estimate also implies the estimate (1) for the limit map $u$. We also observe that in case $l(\gamma)=0$, i.e. if $\rho\left(\{t\} \times S^{1}\right)$ is elliptic or trivial, $u$ will have finite energy, and it will therefore extend through the puncture. As we shall see from our subsequent reasoning, the same will be true in higher dimensions. Therefore, when we consider a nonsingular compactification $\bar{X}$ of $X$ with $D:=\bar{X} \backslash X$ as divisor at infinity, we may enlarge $D$ by adding other divisors $D^{\prime}$ in $\bar{X}$ with nonempty intersection with $X . \rho$ induces a representation $\rho^{\prime}$ of $\pi_{1}\left(X \backslash D^{\prime}\right)$, but the $\rho$-equivariant harmonic map on $X^{\prime} \backslash D$ that we shall construct will then extend as a harmonic map to all of $X$. Enlarging $D$ if necessary, we may therefore assume that $D$ is contained in a linear system $L$, i.e. that $D$ is in the closure of the zero set of a meromorphic function $s$ on $\bar{X}$. For generic $\delta \neq 0$, the level set $|s|=\delta$ is then fibered by nonsingular hypersurfaces $X^{c}=\{z \in X: s(z)=c\}$. By induction hypothesis, we may assume that on each $X^{c}$, we have a pluriharmonic map $u^{c}$ with the correct growth behaviour at infinity. The uniqueness results for harmonic maps also allow one to let these maps depend smoothly on $c$ with $|c|=\delta$. We put $X_{\delta}:=\{z \in X:|s(z)|>\delta\}$ and solve the Dirichlet problem for a harmonic map $u_{\delta}: X_{\delta} \rightarrow N$ with boundary values $\left.u_{\delta}\right|_{X^{c}}=u^{c}$. Although $X_{\delta}$ need not have compact closure in $X$, because $L$ may have base points, one may verify that there exist finite energy maps in the class under consideration, and $u_{\delta}$ may therefore be obtained by minimizing energy.

Lemma. $u_{\delta}$ is pluriharmonic.

For the proof, motivated by [Wd], [NS], [Su2], one integrates Siu's Bochner identity [Su1] over $X_{\delta}$. The integration by parts yields a boundary term which however vanishes because the boundary values are harmonic on the $X^{c}$ and because $\partial X_{\delta}$ is Levi flat. Due to the possible noncompactness of $\partial X_{\delta}$, one also needs an additional cut-off argument in order to exclude divergence of the various integrals involved. Letting $\delta \rightarrow 0$ then produces the desired pluriharmonic $u: X \rightarrow N$.

\section{Strong rigidity of lattices in Hermitian symmetric spaces}

Theorem 2. Let $Y$ be an irreducible Hermitian symmetric space of noncompact type other than the hyperbolic plane. Let $\Gamma$ be a lattice in $Y$. Let $X$ be a smooth quasiprojective variety with contractible universal cover and 
with $\pi_{1}(X)$ isometric to $\Gamma$. Then the universal cover $\tilde{X}$ is \pm biholomorphically equivalent to $Y$, and $\pi_{1}(X)$ is conjugate to $\Gamma$ as a group of automorphisms of $Y$.

If $X$ and $Y / \Gamma$ are compact, this was shown by Siu [Su1]. In the general case, it was shown by Jost-Yau [JY2], [JY3] with an additional technical assumption on a compactification $\bar{X}$ of $X$ needed to ensure the existence of a finite energy map. Theorem 2 is proved with the help of the pluriharmonic map $u$ of Theorem 1 and some Schwarz lemma type arguments.

Theorem 2 generalizes Mostow-Margulis superrigidity in the Hermitian case for archimedean representations. Since Theorem 1 also holds for Tits buildings as image, nonarchimedean representations can be treated as well. This point will be discussed in the next $\S$.

\section{Representations of $\pi_{1}$ of algebraic varieties}

Let $G \subset \operatorname{Sl}(r, K)$ be a simple linear algebraic group over the ground field $K, R(G):=\left\{\rho: \pi_{1}(X) \rightarrow G\right\}$ the set of representations, $M_{B}(G):=$ $R(G) / G$ the moduli space, where $G$ acts by conjugation. For $\alpha \in \pi_{1}(X)$, $\rho(\alpha)$ can be considered as a matrix over $K$, and so it has a characteristic polynomial. Fixing the characteristic polynomials for short loops around the components of the divisor $D:=\bar{X} \backslash X$ at infinity leads to a natural stratification of the affine variety $M_{B}(G)$.

As in [Z1], [Z2], [JZ1], [JZ2], we may deduce rather strong factorization properties for representations that are not rigid or bounded.

Theorem 3. Let $G$ be a simple algebraic group over $\mathbb{C}$, and let $\rho \in M_{B}(G)$ represent a Zariski dense representation. Then either $\rho$ is rigid in the class of representations with the same characteristic polynomials at infinity (as described above), or $\rho$ factors through a holomorphic morphism $f$ : $X \rightarrow Y$ for some quasiprojective $Y$ with $\operatorname{dim} Y \leq \operatorname{rk}_{\mathbb{C}} G$, i.e. there exists a representation $\sigma: \pi_{1}(Y) \rightarrow G$ with $\rho=\sigma \circ f_{\#}$.

Theorem 4. Let $G$ be a simple algebraic group over a complete field $K$ with a discrete valuation. Suppose that the associated Euclidean Tits building $\Delta(G(K))$ is locally compact. Let $\rho: \pi_{1}(X) \rightarrow G$ be Zariski dense. Then either $\rho$ is bounded, i.e. $\rho\left(\pi_{1}(X)\right)$ is contained in a compact subgroup of $G$, or $\rho$ factors through a morphism $f: X \rightarrow Y$ with $\operatorname{dim} Y \leq \operatorname{rk}_{K}(G)$ as in Theorem 3.

The proofs use the pluriharmonic map $u$ of Theorem 1 and the holomorphic fibration associated to it, as well as the constructions of [JZ2], in particular the following Lefschetz type result: 
Lemma. Let $\left\{\omega_{1}, \ldots \omega_{r}\right\}$ be a set of linearly independent holomorphic 1forms with at most log-poles at $\infty$. Then either for some $1 \leq k \leq r$, $k$ linearly independent forms in the span of $\omega_{1}, \ldots \omega_{r}$ factor through a surjective morphism $f: X \rightarrow Y$ with $\operatorname{dim} Y \leq k$, or the Albanese map defined by $\omega_{1}, \ldots \omega_{r}$ lifts to a map $g: \tilde{X} \rightarrow \mathbb{C}^{r}$ with connected fibres. (For $r=1$, this result is due to Simpson [S2].)

\section{Acknowledgement}

This paper partly grew out of a discussion with Peter Kronheimer, and we thank him for his suggestions.

\section{References}

[GS] M. Gromov and R. Schoen, Harmonic maps into singular spaces and p-adic superrigidity for lattices in groups of rank one, Publ. Math. IHES. 76 (1992), $165-246$.

[I] S. Iitaka, Logarithmic forms of algebraic varieties, J. Fac. Sci., University Tokyo, Sec. 1A, 23 (1976), 525-544.

[JY1] J. Jost and S. T. Yau, Harmonic mappings and Kähler manifolds, Math. Ann. 262 (1983), 145-166.

[JY2] - The strong rigidity of locally symmetric complex manifolds of rank one and finite volume, Math. Ann. 271 (1985), 143-152.

[JY3] _ On the rigidity of certain discrete groups and algebraic varieties, Math. Ann. 278 (1987), 481-496.

[JY4] , Harmonic maps and group representations, in: B. Lawson and K. Tenenblat (eds.), Differential Geometry and Minimal Submanifolds Longman Scientific, 1991, 241-260.

[JZ1] J. Jost and K. Zuo, Harmonic maps and $\mathrm{Sl}(r, \mathbb{C})$-representations of $\pi_{1}$ of quasi projective manifolds, J. Alg. Geom., to appear.

[JZ2] - Harmonic maps into Tits buildings and factorization of non rigid and non arithmetic representations of $\pi_{1}$ of algebraic varieties, preprint 1993.

[JZ3] _ Harmonic maps of infinite energy and rigidity results for archimedean and nonarchimedean representation of fundamental groups of quasiprojective varieties, preprint 1994.

[Lk] J. Lohkamp, An existence theorem for harmonic maps, Manuscr. math. 67 (1990), 21-23.

[NS] S. Nishikawa and K. Shiga, On the holomorphic equivalence of bounded domains in complete Kähler manifolds of nonpositive curvature.

[S1] C. Simpson, harmonic bundles on noncompact curves, J. AMS. 3 (1990), 713769.

[S2] _ A Lefschetz theorem for $\pi_{0}$ of the integral leaves of a holomorphic oneform, Compos. Math. 87 (1993), 99-113.

[Su1] Y. T. Siu, The complex analyticity of harmonic maps and the strong rigidity of compact Kähler manifolds, Ann. Math. 112 (1980), 73-111. 
[Su2] _ Complex analyticity of harmonic maps, vanishing and Lefschetz theorems, J. Diff. Geom. 17 (1982), 55-138.

[W] M. Wolf, Infinite energy harmonic maps and degeneration of hyperbolic surfaces in moduli spaces, J. Diff. Geom. 33 (1991), 487-539.

[Wd] J.C. Wood, An extension theorem for holomorphic mappings, Math. Proc. Cambridge Philos. Soc. 88 (1980), 125-127, and 94 (1983), 189.

[Z1] K. Zuo, Some structure theorems for semi-simple representations of $\pi_{1}$ of algebraic manifolds, Math. Ann. 295 (1993), 365-382.

[Z2] _ Factorization of nonrigid Zariski dense representation of $\pi_{1}$ of projective algebraic manifolds, Inv. math. 118 (1994), 37-46.

Department of Mathematics, Ruhr-Universität Bochum, D-44780 Bochum, GERMAnY

E-mail address: juergen.jost@ruba.rz.ruhr-uni-bochum.de

FB Math., Universität Kaiserslautern, D-67653 Kaiserslautern

E-mail address: zuo@mathematik.uni-kz.de 